\title{
Research on Face Expression Recognition
}

\author{
S.Nithya Roopa
}

\begin{abstract}
Face Expression Recognition (FER) has become main area of interest due to its wide applications. Automatic Facial expression recognition has drawn the attention of researchers as it has many applications. Facial Expression Recognition gives important information about emotions of a human being. Many feature selection methods have been developed for identification of expressions from still images and real time videos. This work gives a detailed review of research works done in the field of facial expression identification and various methodologies implemented for facial expression recognition.
\end{abstract}

Keywords-Emotion recognition; automatic emotion recognition; deep learning; image recognition; speech technology; signal processing;

\section{INTRODUCTION}

The most predominant non-verbal communication method used to understand the mentality of a human being is the facial expression. Automatic Facial Expression has become a prime area of research because of its wide applications in various fields like Implementation of Security, Counseling Systems, Lie detection in interrogation etc. A facial expression recognition system is an automated system which can analyze the features of the face from static image or a live video dataset and identify the facial expression into various classes. The expression of the face is a primary form of mental and emotional state. Research of Psychologist Mehrabian [1] shows that only $7 \%$ of the actual information is transmitted orally, and $38 \%$ is passes by auxiliary of language, such as the rhythm and speed of speech, tone, etc. The information ratio which is transmitted by the expression of face has reached $55 \%$. So, most of valuable information can be obtained by facial expression recognition that gives Darwin [2] announced the expression consistency. According to him, the expression of face cannot be estimated by the gender and race of the person. In the 1970's, Ekman and Friesen [3, 4] made a research work for recognizing the facial expression and they defined six basic categories of facial expressions: sadness ,happiness, fear, surprise, disgust and anger which has helped for the research of current expression recognition. In 1991, Maze and Pentland proposed a new concept using optical flow method for the recognition of facial expression [5].

The facial expression recognition is done in various phases. Each stage is discussed in detail as follows

\footnotetext{
Revised Manuscript Received on July 18, 2019.

S.Nithya Roopa, Assistant Professor Kumaraguru College of Technology Coimbatore,Tamilnadu,

Nithyaroopa.s.cse@kct.ac.in)
} the best way to understand person's mental state. In 1872,

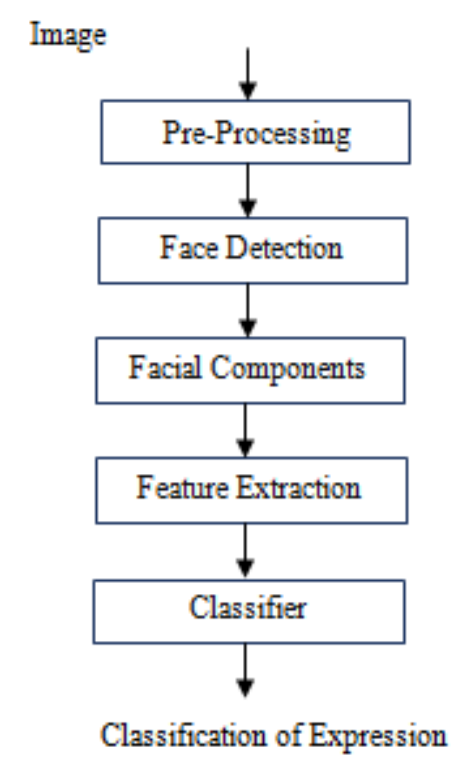

The Automatic Facial Emotion Recognition is done in five stages as shown in Fig.

The removal of noise is done in the initial stage by giving image as an input and gives the face for next processing. The detection of facial components identifies the parts of the individual parts of the face for recognizing the expression. The most common feature selection techniques are Principal Component Analysis, Independent Component Analysis, Local Binary Patterns, Linear Discriminant Analysis, Local Directional Pattern and Local Gradient Code. In the next step, the features are classified into the respective facial expression base classes based on the methods of classification. section 2 and 3 shows some feature extraction techniques. The paper is concluded in section 4 .

\section{BACKGROUND INFORMATION \& RESULTS}

Wang, Xin; Liu, Xingang; metallic element, Longyan; Shen, Zhilin et al. [18], have introduced a replacement Facial feeling Recognition system. It uses the active form mode (ASM) algorithmic program to align the components of the face, then native binary patterns ar extracted and it uses support vector machine (SVM) classifier to spot the feeling of face from the facial expressions extracted.

Kumar, V.; Basha, A.S.A.et al. [17] have introduced a replacement model to recognition of face expression, that uses ripple to reduce the high dimensional knowledge of image spectrum of face expression into an occasional dimension knowledge so uses KNN to classify the 
expressions from the still image.

Happy, S. L; Routray, A. et al. [14] have developed a way for the popularity of expression by utilizing the physical options of facial frames. some primary facial frames, supported the facial landmark's position, was elect that ar active throughout feeling infusion. These active components ar once more refined to retrieve the necessary frames that consists of options for classification of every combine of expressions, extracting totally different facial components as pertinent for various combine of expression categories. Oneagainst-one classification approach is enforced mistreatment these options. The physical options from these components ar given to a multi-class classifier to classify the pictures.

Singh, M.; Majumder, A.; Behera, L.et al. [9] have projected AN automatic facial expressions recognition methodology mistreatment Bayesian network. they need trained the model mistreatment probabilistic modeling that extends relationship between facial expression, action units and finally acknowledges the specified emotions. they need additionally given ways for options extraction to induce spacial feature vector and physical feature vector containing moments extracted when applying Dennis Gabor filter over facial patches.

Majumder, A.; Behera, L.; Subramanian, V.K. et al. [1], have proposed a physical feature based facial expression recognition system using KSOM. Physical features are selected using Local binary patterns (LBPs). The dimensionality of the LBP feature vector was compressed using PCA to dispose the redundant data that leads to additional computation cost. Automatic Facial expression analysis systems identifies expressions of face depends on the selected features. One of the recognition methods is Frame based. Frame based method does not use physical information of the input images. It uses the current input image information with/without a reference frame. The input image can be still image or real time video which can be a stream of images. The other method is sequence based. Sequence based expression uses the physical information of the frames to identify the expression of one or more patches.

Kai-Tai Song; Chao-Yu statue maker et al. [3], planned a physical strengthened approach to develop feeling recognition from the pictures of face. form and material models of pictures of faces were processed by victimization active look model (AAM), from that facial feature points and physical feature values were elect. the chosen options were employed by connectedness vector machine (RVM) to spot emotional states. they need given a temporal analysis technique for distinctive the emotional categories, specified the factors square measure associated with the relevant class fitly.

Jizheng Yi; Xia Mao; Lijiang Chen; Yuli Xue; Compare, A. et al. [2] have given a completely unique Facial feeling Recognition algorithmic program by utilizing the physical characteristics and therefore the texture info of the pictures. The feature points were identifying by a full of life look model. Then, 3 countenance, that square measure distance magnitude relation constant of feature points, magnitude relation constant of affiliation angle and energy parameter of skin deformation, were applied to scale back the variations among the people. Finally, a radial basis operate neural network was exploited because the classifier for the algorithmic program.

Li Xia et al. [5] developed the feeling identification technique supported SVM for the failings of the normal classification ways. It identifies quick classification with a mixture of sub-classifier, reducing the classification error. analysis shows that the multiclassification technique supported SVM will bring down the coaching and testing time and improve the performance of classification.

Myunghoon Suk; Prabhakaran, B. et al. [8] have planned a model victimization the Support Vector Machines(SVMs) for classifying the emotions. The options of \{facial expression| countenance facial options face expression $\}$ for recognizing the emotions were elect by Active form Model (ASM) setting the landmarks on a face so connected options were created by the displacement between neutral and expression features.

$\mathrm{Mu}-\mathrm{Chun} \mathrm{Su}$, Chun-Kai principle, Shih-Chieh architect, De-Yuan Huang, Yi-Zeng Hsieh, and Pa-Chun Wang et al. [10], have developed AN automatic countenance recognition system supported feature maps which may be selforganizing. Viola and Jones were accustomed differentiate a face from a complete image. when an individual's face is known, a composite methodology was bestowed to find pupils so the detected face image will be cut, rotated. At last, a multi-layer perceptron (MLP) was enforced for the classification of the expressions.

Urvashi Bakshi, Rohit Singhal [16], have projected a brand-new methodology to spot external body part victimization DCT, PCA and Kyrgyzstani monetary unit neural network. PCA may be a ancient methodology of reducing dimension. distinct circular function remodel (DCT) may be a fashionable compression technique and Self Organize Map (SOM) is employed to classify the suitable emotions.

Lisai Li; Zilu Ying; Tairen principle et al. [4] have introduced AN rule for facial \{features face expression\} Recognition (FER) that depends on fusion of Gabor texture features and native part quantization (LPQ). The LPQ feature and Gabor texture feature were elect from the expression image sequence. LPQ options ar born-again into histograms of LPQ remodel. 
TABLE 1

EMOTIONAL DATASET

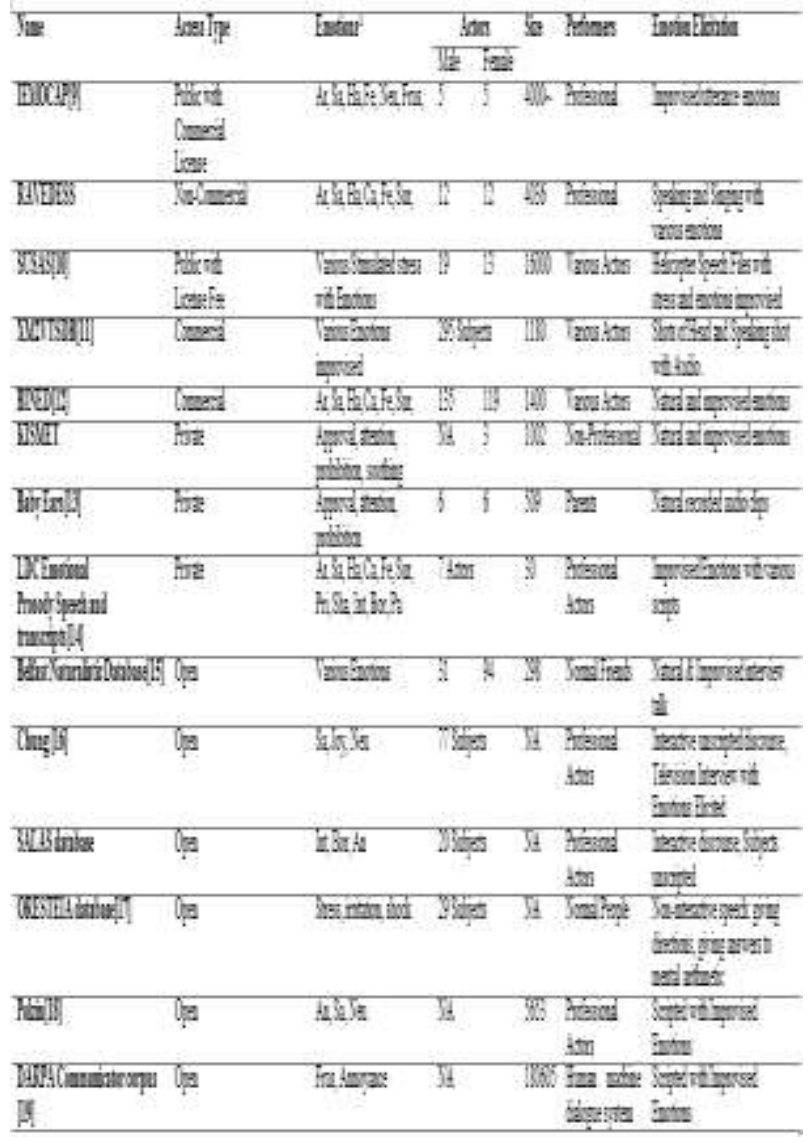

\section{CONCLUSION}

Facial Expression recognition has wide application areas and it needs additional correct and reliable FER system. This work has given a survey on face expression recognition techniques. The feature extraction ways area unit exploited together with comparison. in line with the survey varied approaches area unit out there for establish face expression. during this paper numerous face expression recognition techniques, its associated areas and numerous analysis challenges is being surveyed. Methods with higher recognition rate have sensible performance These techniques give a temporal answer to the face expression recognition downside. feeling detection from face expression is a problem that causes difficulties. So, analysis during this space can continue for years as several solutions needs to be to make a perfect programmed. A comparative study is completed with numerous image information bases by differentiating the options.

\section{REFERENCES}

1. Tuark MA, Peintland AP. feeling recognition mistreatment eigenfaces. In: laptop Vision and Pattern Recognition. Proceedings,IEEE laptop Society Conference on. IEEE; 1991, p. 586-91.

2. M. El Ayiadi, M. S. Kaamel, and F. Kaerray, "Survey on feeling recognition: options, classification schemes, and information," Pattern Recognit., vol. 44, no. 3, pp. 572587, 2011.

3. Y. Leicun, Y. Beingeo, and G. Henton, "Deep learning," Nature, vol. 521, no. 7553, pp. 436-444, 2015.
4. J. Schmedhuber, "Deep Learning in convolutional neural networks: an summary," Neural Networks, vol. 61, pp. 85-117, 2015.

5. J. Nagiam, A. Khoesla, M. Kaim, J. Naim, H. Leei, and A. Y. Nig, "Multimodal Aeriel Deep Learning," Proc. 28th Int. Conf. Mach. Learn., pp. 689-696, 2011.

6. F. Deipl and T. Voigt, "Automatic feeling recognition from Expression," 2010.

7. S. Lugovec, I. Dinder, and M. Hoervat, "Approaches and applications of feeling recognition," 2016 thirty ninth Int. Conv. Inf. Commun. Technol. Electron. Microelectron. MIPRO 2016 - Proc., no. November 2017, pp. 1278 1283, 2016.

8. B. Schuller, G. Rigoll, and M. Lang, "Emotion recognition combining Physical options and linguistic info during a hybrid support vector machine - belief specification," Acoust. Speech, Signal method., vol. 1, pp. 577-580, 2004.

9. C. Bussieo ett ail., "IEMOCAP: Interactive emotional dynamic motion capture information," Laing. Resoer. Evail., vol. 42, no. 4, pp. 335-359, 2008.

10. J. H. Haensen, S. E. Boiu-Ghaizale, R. Sarikaaya, and B. Pelliom, "Getting Started with SUSAS: A Speech underneath Simulated and Actual Stress information," Eurospeech, pp. 1743-46, 1997.

11. K.Meisser; J.Mataes; J.Keittler;K., "XM2VTSDB: The Extended M2VTS information."

12. I. Sneiddon, M. Mc.Rorrie, G. MicKeown, and J. Hanratty, "The iatrogenic natural feeling information for detection," IEEE Trans. Affect. Comput., vol. 3, no. 1, pp. 32-41, 2012.

13. M. Slaeney, "BabyEars: A recognition system for emotive vocalizations," Speech Commun., vol. 39, no. 34, pp. 367-384, 2003.

14. K. D. Gairett et al., "Correlates within the Comprehension of Emotional Prosody," no. February, p. 19104, 2002.

15. E. Douglaas-cowiee, R. Coweie, and M. Schröoder, "A New feeling Database: concerns, Sources and Scope," In, pp. 39-44, 2000.

16. D.Erickson, "Expressive: Production, perception and application to synthesis," Acoust. Sci. Technol., vol. 26, no. 4, pp. 317-325, 2005.

17. S. McGilloway, R. Cowie, E. Douglas-Cowie, S. Gielen, M. Westerdijk, and S. Stroeve, "Approaching automatic recognition of feeling from Voice: A rough benchmark," Proc. ISCA Work. Speech Emot., pp. 207-212, 2000.

18. T. S. Polzein and A. Waeibel, "Emotion-Sensitive Human-Computer Interfaces," ISCA Tutor. Res. Work. Speech Emot., 2000.

19. C. aeronaut and A. Rudnicky, "The Carnegie altruist soul Corpus," Proc. Int. Conf. Spok. Lang. Process., no. August, pp. 341-344, 2002.

20. U. Shrawankar and V. M. Thakare, "Techniques for Feature Extraction In Speech Recognition System : A Comparative Study," 2013.

21. Z. Fang and Z. Guoliang, "Comparison of various I $\mathrm{m} \mathrm{p}$ e m e n t a ti o n s of M F C C," vol. 16, no. 6, pp. 2-3, 2001.

22. I. Miporas and T. Ganchiev, "Comparison of speech options on the speech recognition task," J. Comput. ..., vol. 3, no. 8, pp. 608-616, 2007.

23. D. O'Shaugihnessy, "Linear prophetical cryptography.," IEEE Potentials, vol. 7, no. 1, pp. 29-32, 1988.

24. J. Dae Wiu and B. F. Lein, "Speaker identification supported the frame linear prophetical cryptography spectrum technique," professional Syst. Appl., vol. 36, 
no. 4, pp. 8056-8063, 2009.

25. C. K. Rusehforth, "Emotion Detection mistreatment Linear prophetical cryptography," IEEE Trans. Acoust., vol. 25 , no. 5, pp. 361-367, 1977.

26. S. Suiny, D. P. Shaw., and K. P. Jacab, "Feature Extraction ways supported linear prophetical cryptography and riffle Packet Decomposition," 2012 Int. Conf. Adv. Comput. Commun., pp. 27-30, 2012.

27. H. Hermensky and L. A. Coex, "Linear prophetical (PLP) Analysis-Resynthesis Technique," vol. 87, no. 303, pp. 5-6, 2013.

28. H. Hermensky, "Perceptual linear prophetical (PLP) analysis," J. Acoust. Soc. Am., vol. 87, no. 4, pp. 1738 $1752,1990$.

29. C. Kurien and K. Balekrishnan, "Linear prophetical cepstral constant isolated digit recognition," Commun. Comput. Inf. Sci., vol. 204 CCIS, pp. 534-541, 2011.

30. V. Sugendhi, "Spectral Analysis in Speech process Techniques," vol. 3, no. 1, pp. 74-76, 2013.

31. T. Seehapich and S. Woengthanavasu, "Emotion Recognition mistreatment Support Vector Machines," 2013 fifth Int. Conf. Knowl. good Technol., pp. 86-91, 2013.

32. Y.-L. L. Y.-L. Lein and G. W. G. Weei, "Speech feeling recognition supported HMM and SVM," 2005 Int. Conf. Mach. Learn. Cybern., vol. 8, no. August, pp. 18-21, 2005.

33. S. Laetif, R. Rena, S. Youinis, J. Qaadir, and J. Eipps, "Cross Corpus Speech feeling Classification- an efficient Transfer Learning Technique," 2018.

34. Y. Huiang, M. Hiu, X. Yeu, and T. Waing, "Pattern Recognition," vol. 663, pp. 721-729, 2016.

35. J. Gidion, S. Khorraem, Z. Aladeneh, D. Dimitridis, and E. M. Provest, "Neural networks for transfer learning in feeling recognition," Proc. Annu. Conf. Int. Speech Commun. Assoc. INTERSPEECH, vol. 2017-Augus, pp. 1098-1102, 2017.

36. A. Balekrishnan and A. Reige, "Recognizing Emotions from Speech mistreatment Deep Neural Networks," 2017.

37. J. Chaing and S. Scheireir, "Learning representations of emotional speech with deep convolutional generative adversarial networks," ICASSP, IEEE Int. Conf. Acoust. Speech Signal method. - Proc., pp. 2746-2750, 2017. 